\title{
Joint Estimation of Impairments for OFDM and MIMO OFDM Systems
}

\author{
T. Shankar \\ VIT University \\ SENSE, VIT University, \\ Vellore, India
}

\author{
A. Karthikeyan \\ VIT University \\ SENSE, VIT University, \\ Vellore, India
}

\author{
Christopher Clement $J$ \\ VIT University \\ SENSE, VIT University, \\ Vellore, India
}

\begin{abstract}
Orthogonal Frequency Division Multiplexing (OFDM) is a multi-channel modulation technique that makes use of Frequency division multiplexing (FDM) being modulated by a low bit rate digital stream. The main reason of using OFDM is because here the symbol detection is easy and also to increase the robustness against frequency selective fading and narrow band interference. Certain parameter like CFO (carrier frequency offset),I/Q imbalance (inphase and quadrature phase imbalance) imbalance causes distortion in the received signal. Here comparison is made for the given parameters using different channels and a method is proposed for Multiple Input Multiple output (MIMO) OFDM system when symbol generated is not a integral multiple of number of transmitters used.
\end{abstract}

Key Words: OFDM, FDM, CFO, I/Q Imbalance, MIMO OFDM.

\section{INTRODUCTION}

OFDM technique has now days become of great importance because of its simplicity and robustness in the field of communication. For correct detection of received symbol at the receiver certain parameters like $\mathrm{CFO}$ (carrier frequency offset), I/Q imbalance (inphase and quadrature phase imbalance) and channel response has to be known, when these parameters are not properly estimated it can affect the performance of the system.

CFO is mainly caused due to mismatch of carrier frequency of oscillator at the transmitter and receiver this results in ICI and increase in BER. I/Q imbalance is caused by mismatch of oscillator at the receiver and transmitter thus affecting the orthogonality of subcarriers in the system. In this paper MSE of CFO and I/Q imbalance are being compared for different channels (awgn, Rayleigh, rician) for OFDM using the concept of Channel Residual energy (CRE)[1]. In case of MIMO OFDM the concept of CRE[1] has been extended to estimate the impairments when number of transmitted symbol is not an integral multiple of number of transmitter.

\section{SYSTEM MODEL}

We consider an OFDM system where $\mathrm{M}$ is the number of symbol used. We first take the $\mathrm{M}$-point 1DFT to obtain an M $\mathrm{x}$ 1 vector $\mathrm{x}$ and then a Cyclic Prefix $(\mathrm{CP})$ of length $\mathrm{L}$ is appended before transmitting it through the channel. Now considering the
I/Q imbalances and $\mathrm{CFO}$, the transmitter and receiver I/Q parameters are defined as

$$
\mu_{i}=\frac{1+\varepsilon_{i} e^{-j \phi_{i}}}{2}, v_{i}=\frac{1-\varepsilon_{i} e^{j \phi_{i}}}{2}, \text { for } \mathrm{i}=(\mathrm{t}, \mathrm{r})
$$

where $t$ and $r$ stand for transmitter and receiver respectively, and the quantities $\phi$ and $\varepsilon$ are respectively the phase and the amplitude mismatch of the oscillators.

The received vector due to the I/Q imbalance and cfo is given by $\tilde{r}=\mu_{r} E r+v_{r}(E r) *$

where

$\mathrm{E}=\operatorname{diag}\left[1 \quad e \frac{j 2 \pi \theta}{M} \quad . . e \frac{j 2 \pi(M-1) \theta}{M}\right]$,

and

$r=H_{c i r}\left(\mu_{t} x+v_{t} x^{*}\right)+q,-$

where $\mathrm{q}$ is the channel noise and $\theta$ is the normalized CFO.

\section{ALGORITHM:}

Defining the transmitter and receiver i/q parameter as

$\alpha_{t}=\frac{v_{t}}{\mu_{t}}, \alpha_{r}=\frac{v_{r}}{\mu_{r}}$,

the channel estimate is given by

$\tilde{h}_{0}=\frac{i n v\left(X_{c i r}+\alpha_{t} X_{c i r} *\right) E^{*} r^{*}\left(\tilde{r}-\alpha_{r} \tilde{r}^{*}\right)}{1-\left|\alpha_{r}\right|^{2}}$

Where $\tilde{h}_{0}$ is a $\mathrm{M} \times 1$ vector.

We require the first $(\mathrm{L}+1)$ terms for the channel response and we need to minimize the energy of the last (M-L-1) terms. CRE is the energy of these (M-L-1) terms which need to be minimized and it is a function of $\alpha_{r}, \alpha_{t} \theta$ So in order to minimize the value of CRE we have to find the optimum value of $\alpha_{r}, \alpha_{t}, \theta$ using the method proposed in[1].

We define the matrices $\mathrm{A}$ and $\mathrm{B}$ as

$A=X_{c i r}^{-1} E *$ and $B=X_{c i r}^{-1} X_{c i r} * X_{c i r}^{-1} E *$

And also define a matrix $\phi$ where $\phi$ is a 
(M-L-1) x 2 matrix as

$\left[\begin{array}{c}\alpha_{r,}, o p t \\ \alpha_{t}, o p t\end{array}\right]=\phi^{+} \phi^{-1} \phi^{+} A \tilde{r}$

Where $\alpha_{r}$,opt and $\alpha_{t}$ opt are the optimal value of the $\alpha_{r}$ and $\alpha_{t}$ respectively.

CRE is a function of $\theta$ hence it can be written as

$C R E(\theta)=\left\|A \tilde{r}-\alpha_{r, o p t} A \tilde{r}^{*}-\alpha_{t, o p t} B \tilde{r} \quad+\alpha_{t, o p t} \alpha_{r, o p t} B \tilde{r} *\right\| \wedge 2$

And the optimal value is given by

$\theta_{\text {opt }}=\arg \min _{\theta} \operatorname{CRE}(\theta)$

After finding the optimal value of these impairments the corresponding mean square error value for $\mathrm{CFO}, \mathrm{I} / \mathrm{Q}$ imbalance and channel response is calculated and the graphs are plotted for them.

\section{MIMO OFDM}

Here we calculate the impairment for the MIMO OFDM [2] system using the concept used for OFDM[1] for the different cases:

Case 1: When $\mathrm{M}$ is a multiple of $\mathrm{Nt}$

This case is similar to the case of OFDM system

In this case when the number of transmitter antenna is $\mathrm{Nt}$ we will assume that there are total of $\left(\mathrm{Nt}^{*} \mathrm{~L}\right)$ channel response. Hence here CRE will be the energy of the (M-(Nt*L)) terms of the channel estimate. Considering the case for $\mathrm{Nt}=2$ transmitter antenna. In this case the energy of first $L$ terms and $(M / 2)$ to $(\mathrm{M} / 2+\mathrm{L})$ terms will contribute to the channel response and the CRE will be energy of the $(\mathrm{L}+1)$ terms to $(\mathrm{M} / 2)$ terms and $(M / 2+L)$ terms to the $(M)$ terms. Calculation of MSE for $\mathrm{CFO}$ and I/Q imbalance can be calculated similar to OFDM.

CASE 2: When $\mathrm{M}$ is not a multiple of $\mathrm{Nt}$

If $\mathrm{M}$ is not a multiple of $\mathrm{Nt}$ we can append (a-L) zeros (where a is the largest number $<=\mathrm{M} / \mathrm{Nt}$ ) to the first $\mathrm{Nt}-1$ transmitter antennas and $\mathrm{M}-(\mathrm{Nt}-\mathrm{a})$ zeros to the last transmitter antennas, hence the joint estimation method can be applied using the concept used for OFDM.

\section{SIMULATION RESULTS}

The DFT size is taken as $M=128$ and the length of the channel is taken as $\mathrm{L}=16$. Channel is initially taken as Gaussian and MSE of CFO,

I/Q imbalance and Channel response is calculated. These impairments are later calculated for different channels such as Rayleigh and Rician channel.

Simulation was performed using the parameters as $M=128$, $\mathrm{L}=16, \varepsilon_{t}=1.05, \varepsilon_{r}=1.07$,

$\phi_{t}=5^{\circ}$ and $\phi_{r}=-8^{\circ}$

MSE of CFO, I/Q imbalance and channel response was calculated for both the cases of the MIMO OFDM system for the Gaussian channel and this can be extended for the case of Rayleigh and Rician channel.

\subsection{OFDM Sytem}

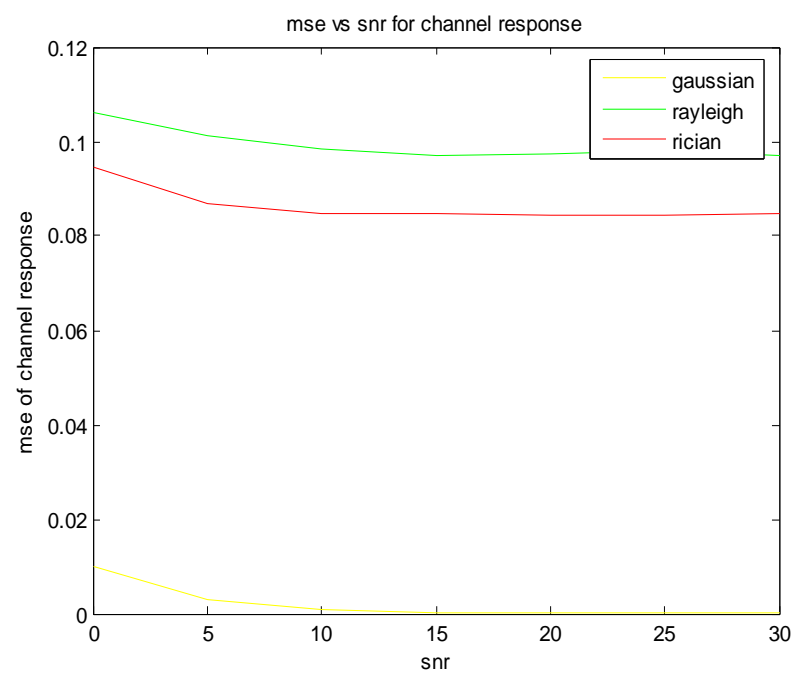

Fig(1) mse vs snr for channel response with the increase in value of snr mse decreases and for gaussain channel having the least mse.

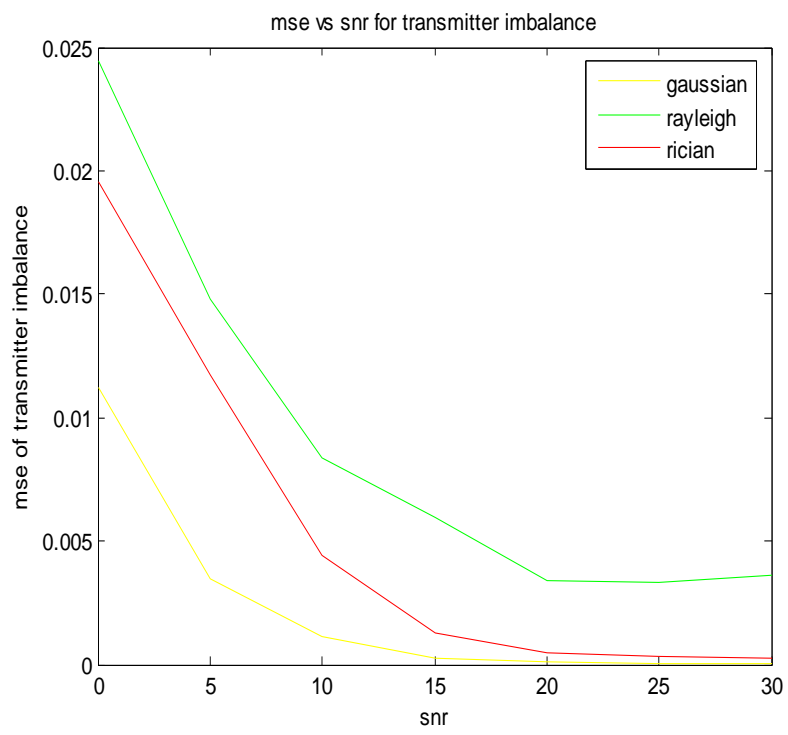

Fig(2) mse vs snr for transmitter imbalance with the increase in value of snr mse decreases and for gaussain channel having the least mse. 


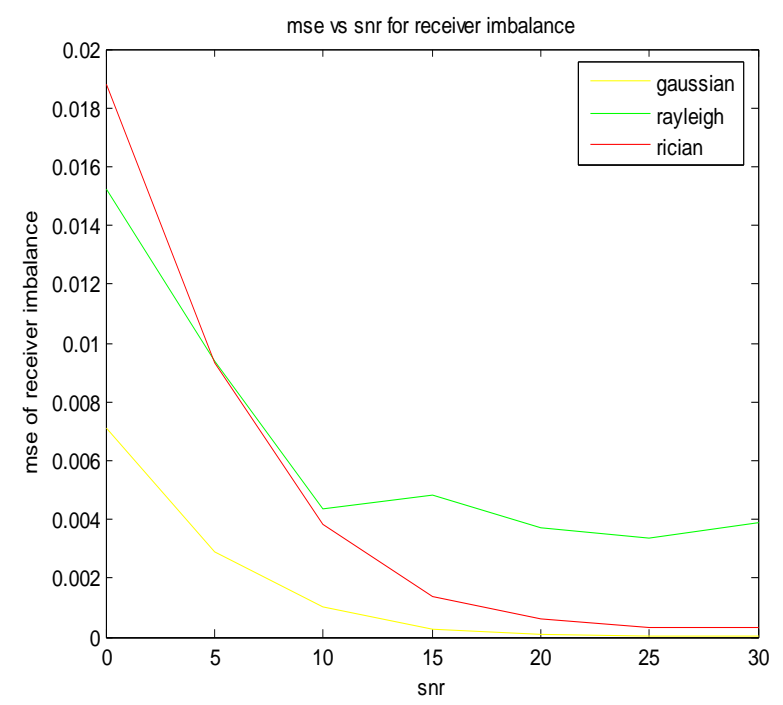

Fig(3) mse vs snr for receiver imbalance, with the increase in value of snr mse decreases and for gaussain channel having the least mse.

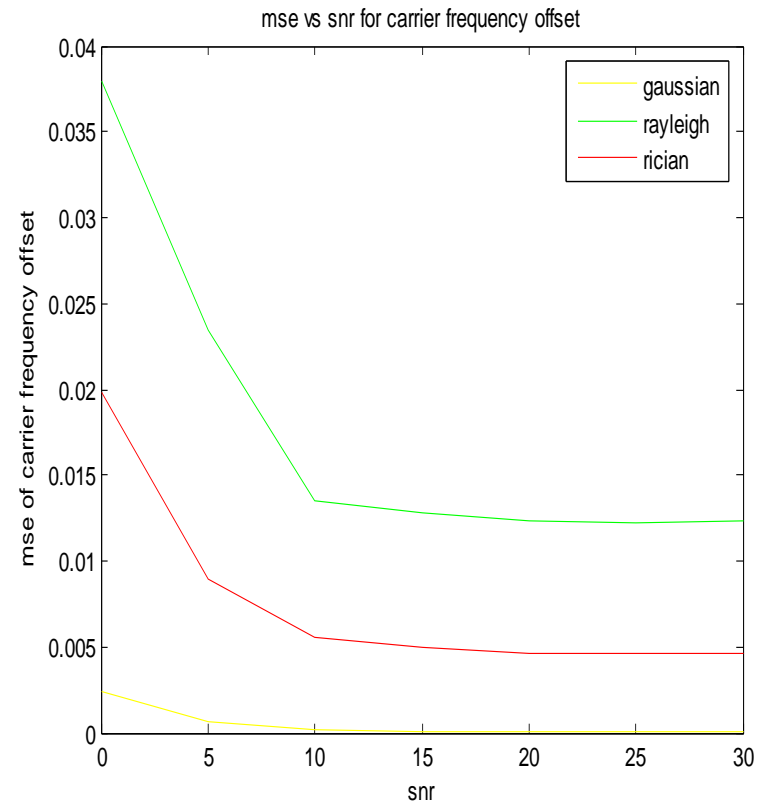

Fig(4) mse vs snr for carrier frequency offset with increase in value of snr mse decreases and for gaussain channel having the least mse.

\subsection{MIMO OFDM System}

Case 1 When M Is A Multiple Of Nt

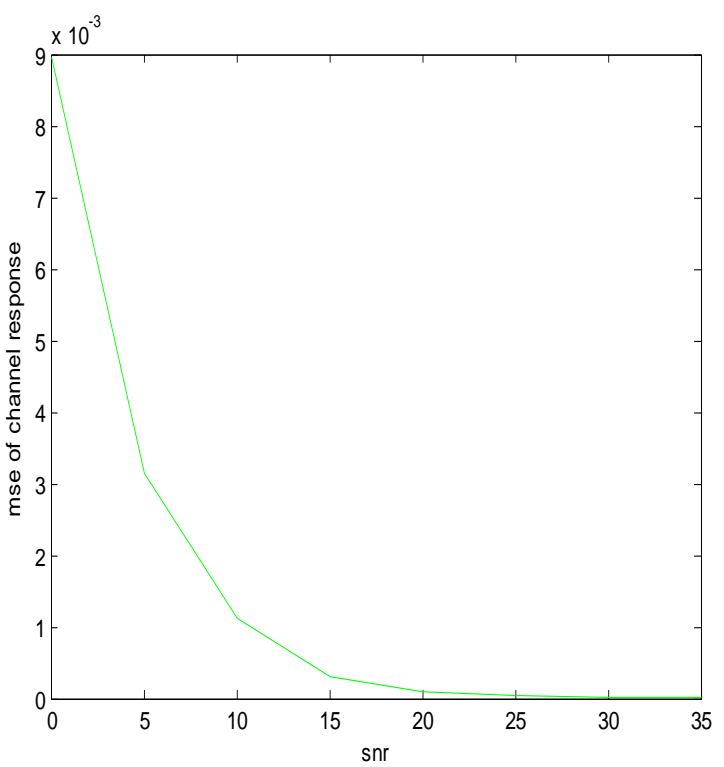

Fig(5) mse vs snr for channel response in gaussian channel,with increase in snr mse decreases

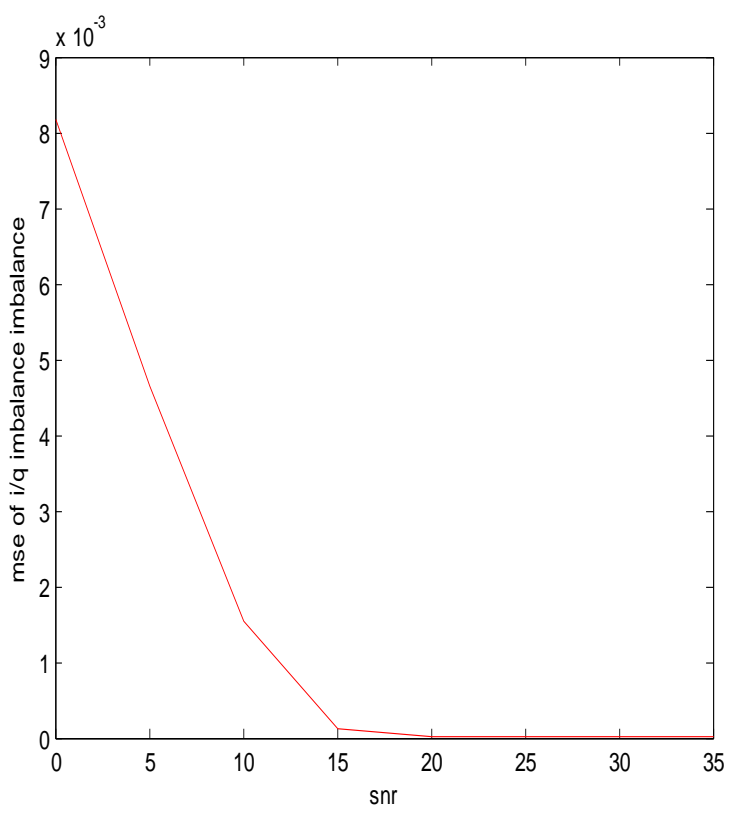

Fig(6) mse vs snr for i/q imbalance in gaussian channel,with increase in snr mse decreases. 


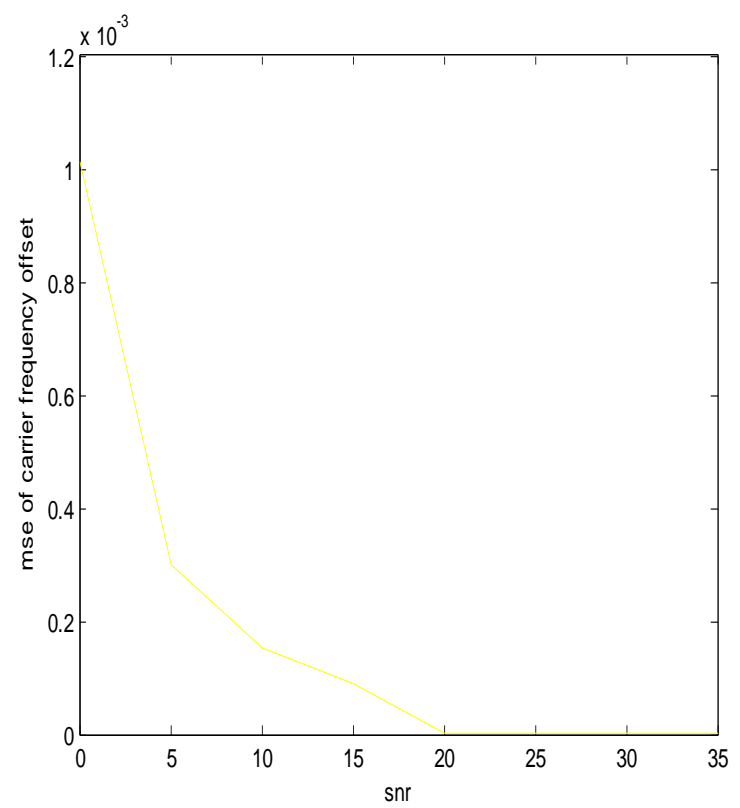

Fig(7) mse vs snr for cfo in gaussian channel With increase in snr mse decreases

\section{CASE 2: When M Is Not Multiple Of Nt}

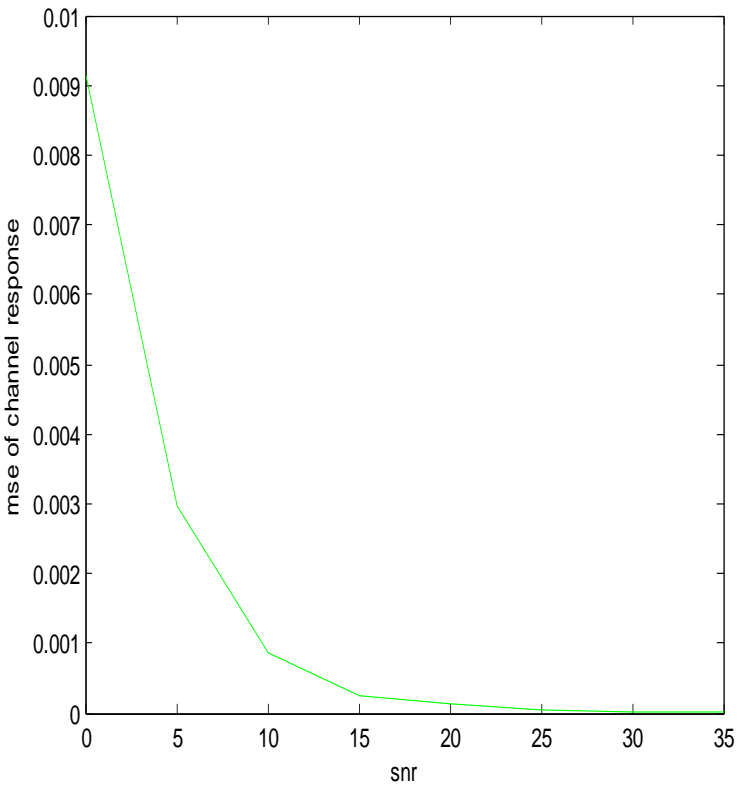

Fig(8) mse vs snr for channel response in gaussian channel,with increase of snr mse decreases.

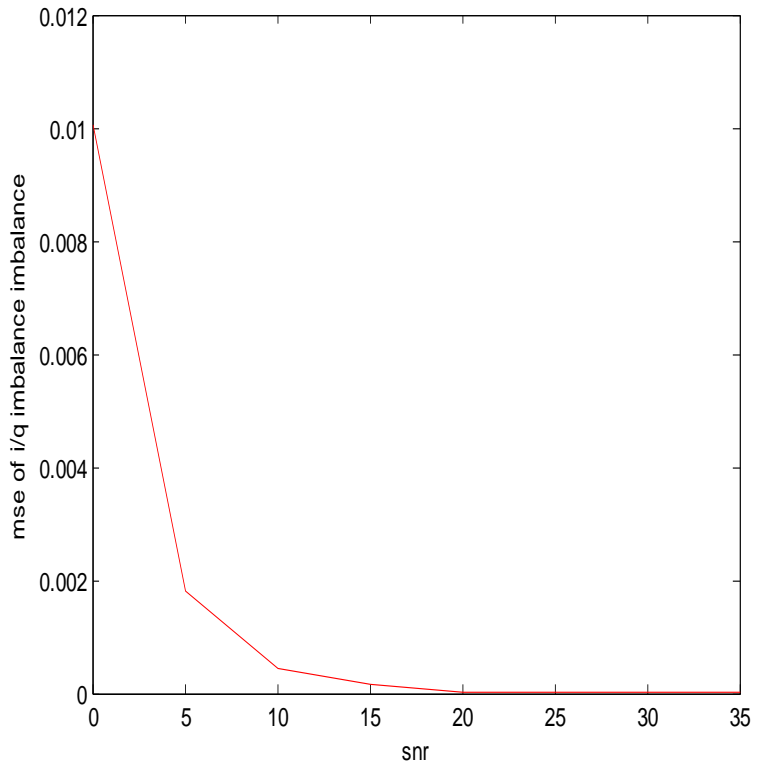

Fig(9) mse vs snr for i/q imbalance in gaussian channel,with increase of snr mse decreases.

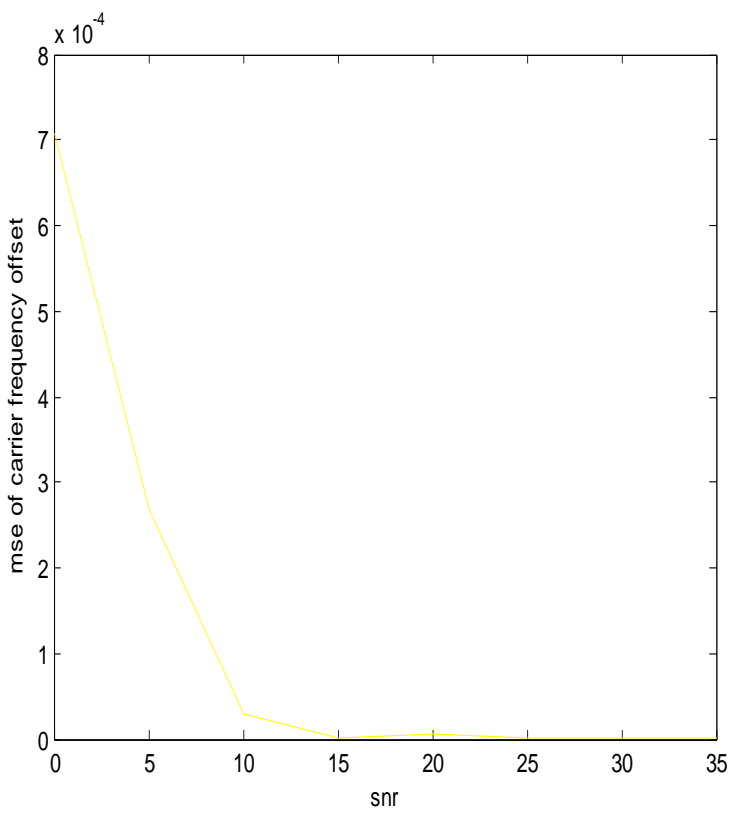

Fig(10) mse vs snr for cfo in gaussian channel,with increase in snr mse decreases 


\section{CONCLUSION}

The joint estimation of MSE of channel response, CFO and I/Q imbalance was determined for different channels for OFDM system and for MIMO OFDM system it was determined for the Gaussian channel. We find that in case for the OFDM system, the mean square error is minimum for the Gaussian channel. We also came to the conclusion that that MSE of the receiver imbalance is less than that of the transmitter imbalance in case of OFDM system.

\section{REFERENCES}

[1] Yuan -hwui chang and see-may phoong "Joint estimation of transmitter and receiver I/Q Imbalances, CFO, and Channel Response for OFDM system".

[2] Yuan -hwui chang and see-may phoong "Joint estimation of I/Q imbalance,CFO and Channel response for MIMO OFDM system"

[3]Y.-H. Chung and S.-M. Phoong, Channel estimation in presence of transmitter and receiver I/Q mismatches in presence of OFDM system.

[4] G. Xing, M. Shen, and H. Lui,"Frequency offset and YQ imbalance compensation for direct-conversion receivers," IEEE Trans.

[5] P. H. Moose,"A technique for frequency division multiplexing offset correction;'IEEE Trans.
[6] T. M. Schmid! and D. C. Cox, "Robust frequency and timing synchronization for OFDM," IEEE Trans. Commun.,

[ 7] F. Horlin, A. Bourdoux, E. Lopez-Estraviz and L. V. der Perre, "Lowcomplexity EM-based joint acquisition of the carrier frequency offset and IQ imbalance,"IEEE Trans. Wireless Commun.

[ 8] P. Stoica and O. Besson, "Training sequence design for frequency offset and frequency-selective channel estimation," 19 10- 19 17, Nov. 2001.

[ 9] y'-H Chung, K.-D. Wu and S.-M. Phoong, "Joint estimation of I/Q imbalance, CFO and channel response for OFDM systems;'

[ 10] G.-T. Gil, I.-H. Sohn, J.-K. Park, and Y. H. Lee, "Joint ML estimation of carrier frequency offset, channel, YQ mismatch, and DC offset in communication receivers,"

[ 11] Q. Zou, A. Tarighat, K. Y. Kim and A. H. Sayeed, "OFDM channel estimation in the presence of frequency offset, IQ imbalance and phase noise," 